Received: 05.02 .2020

Revised: 07.05.2020

Accepted: 19.06 .2020

DOI: $10.17804 / 2410-9908.2020 .3 .061-066$

\title{
APPROXIMATION OF THE CREEP CURVE UP TO THE MOMENT OF NECKING
}

\author{
V. V. Nazarov \\ Research Institute of Mechanics, Lomonosov Moscow State University, \\ 1 Michurinskiy Ave., Moscow, 119192, Russian Federation \\ iD https://orcid.org/0000-0002-9234-3646 $\otimes$ inmec130@mail.ru \\ Corresponding author. E-mail: inmec130@mail.ru \\ Address for correspondence: 1 Michurinskiy Ave., Moscow, 119192, Russian Federation
}

In this paper, we propose a model for describing the creep process up to necking. The specified slope of the tangent to the creep curve is used as a criterion for the appearance of necking. The analysis of the calculated data shows that the proposed model is in satisfactory agreement with the experimental creep curves obtained for the VT5 titanium alloy at $600{ }^{\circ} \mathrm{C}$.

Keywords: necking criterion, creep, uniaxial tension.

\section{References}

1. Nazarov V.V. and Lepeshkin A.R. Features of forming and fracture of rectangular plate with a circular hole. News VolgSTU, Problems of Materials Science, Welding and Mechanical strength, 2016, no. 2, pp. 75-78. (In Russian).

2. Derevyagina L.S., Panin V.E. and Gordienko A.I. Self-organization of plastic shears in localized deformation macrobands in the neck of high-strength polycrystals, its role in material fracture under uniaxial tension. Physical Mesomechanics, 2008, vol. 11, iss. 1-2, pp. 51-62. DOI: 10.1016/j.physme.2008.03.006.

3. Akhmetzyanov M.Kh., Albaut G.N., and Baryshnikov V.N. Study of Stress Strain State in the Neck of Plane Metallic Specimens in Extension by the Method of Photo-Elastic Coverings. Zavodskaya Laboratoriya. Diagnostika Materialov, 2004, vol. 70 (8), pp. 41-51. (In Russian).

4. Lokoshchenko A.M. and Teraud V.V. Analysis of the localization of deformation in cylindrical samples by the photographic registration at the high-temperature creep. Deformatsiya i Razrushenie Materialov, 2013, no. 11, pp. 43-46. (In Russian).

5. Agakhi K.A., Kuznetsov, V.N., Lokoschenko, A.M., Koval'kov V.K., Fomin L.V. Simulation of creep process on the basis of observed data approximation. Mashinostroenie i Inzhenernoe Obrazovanie, 2011, no. 2, pp. 52-57. (In Russian).

6. Koval'kov V.K., Nazarov V.V., Novotnyi S.V. Procedure of high-temperature within complex stressed state. Zavod. Lab. Diagn. Mater., 2006, vol. 72, no. 4, pp. 42-44. (In Russian). 
Подана в журнал: 05.02.2020

УДК 620.172.2:620.162.3:539.376

DOI: $10.17804 / 2410-9908.2020 .3 .061-066$

\title{
АППРОКСИМАЦИЯ КРИВОЙ ПОЛЗУЧЕСТИ ДО МОМЕНТА ВРЕМЕНИ ПОЯВЛЕНИЯ ШЕЙКИ
}

\author{
В. В. Назаров \\ Научно-исследовательский институт механики \\ Федерального государственного бюджетного образовательного учреждения высшего образования \\ «Московский государственный университет им. М.В. Ломоносова» \\ Мичуринский проспект, 1, г. Москва, Российская Федерация \\ (iD https://orcid.org/0000-0002-9234-3646 هinmec130@mail.ru \\ Ответственный автор. Электронная почта: inmec130@ mail.ru \\ Адрес для переписки: Мичуринский проспект, 1, г. Москва, 119192, Российская Федерация
}

В работе предложена модель описания процесса ползучести до момента времени появления шейки. В качестве критерия появления шейки принят заданный наклон касательной к кривой ползучести. Анализ расчетных данных показывает, что предложенная модель удовлетворительно согласуется с экспериментальными кривыми ползучести, полученными для титанового сплава ВТ5 при $600{ }^{\circ} \mathrm{C}$.

Ключевые слова: критерий появления шейки, ползучесть, одноосное растяжение.

\section{1. Введение}

Как правило, механические характеристики материала определяют из результатов испытаний цилиндрических образцов на одноосное растяжение. Различают следующие последовательные стадии процесса деформирования [1]: однородное деформирование (стадия I), удлинение образца с учетом эволюции шейки (стадия II) - в работе эту стадию не рассматриваем. Результаты экспериментального исследования показывают, что эволюция шейки происходит вдоль сопряженных направлений максимальных касательных напряжений [2]. Для расчета напряжений и деформаций области локализации деформирования применяют метод фотоупругих покрытий [3]. В статье [4] приведены результаты экспериментального исследования эволюции шейки при одноосном растяжении цилиндрического образца из алюминиевого сплава Д16Т при $400{ }^{\circ} \mathrm{C}$ (температура плавления равна $650{ }^{\circ} \mathrm{C}$ ). Из анализа фотоснимков и кривой ползучести установлена продолжительность стадии деформирования образца с шейкой, которая оказалась равна 30-40\% от времени в момент разрушения. Эти данные получены при малых временах в момент разрушения, кроме этого, испытания проводились с некоторой неоднородностью температуры на образце, при этом разрушение произошло в верхней концевой точке.

При аппроксимации кривой ползучести разными учеными предложены различные модели описания процесса деформирования без учета эволюции шейки, например [5]. В действительности, к моменту разрушения продольный размер области локализации деформирования может оказаться соизмерим с удлинением образца при однородном деформировании, что может привести к недостаточно точному вычислению значения растягивающего напряжения. Таким образом, чтобы с достаточной точностью описать процесс однородного деформирования, потребуется использовать критерий момента времени появления шейки. 


\section{2. Критерий появления шейки}

Используем обозначения: $\varepsilon(t)$ - зависимость деформации ползучести от времени; $\dot{\varepsilon}(t)$ - зависимость скорости деформации удлинения от времени $\left(\dot{\varepsilon}_{0}=\left.\dot{\varepsilon}\right|_{t=0}\right) ; \sigma(t)-$ зависимость растягивающего напряжения от времени $\left(\sigma_{0}=\left.\sigma\right|_{t=0}\right) ; d(t)$ и $l(t)$ - зависимости диаметра и длины цилиндрического образца от времени $\left(d_{0}=\left.d\right|_{t=0}, l_{0}=\left.l\right|_{t=0}\right) ; s(t)$ - зависимость площади поперечного сечения от времени $\left(s_{0}=\left.s\right|_{t=0}\right) ; F$ - стационарная растягивающая сила; $\dot{\varepsilon}_{*}=\left.\dot{\varepsilon}\right|_{t=t_{*}}-$ скорость деформации удлинения в момент времени $t_{*}$ появления шейки; $t_{* *}-$ время в момент разрушения. Считаем, что в момент времени $t_{*}$ появления шейки происходит резкое возрастание скорости деформации удлинения, что определяется некоторым тангенсом угла $\vartheta$ наклона касательной к кривой ползучести $\varepsilon(t)$, что означает

$$
\dot{\varepsilon}_{*} \cdot t_{* *}=\operatorname{tg}(\vartheta), \quad 0<\vartheta<\pi / 2 \text {. }
$$

Будем использовать среднее значение $\vartheta=\pi / 4$, при котором $\operatorname{tg}(\vartheta)=1$. Из равенства (1) находим значение $\dot{\varepsilon}_{*}=t_{* *}^{-1}\left(t_{* *}-\right.$ измерено в эксперименте). Из диаграммы $\dot{\varepsilon}(t)$ (соответствует экспериментальным данным $\varepsilon(t))$ находим значения $t_{*}$ и $\varepsilon_{*}$ - предельные значения аппроксимации кривой ползучести.

\section{3. Модель описания процесса однородного деформирования}

Уравнение механического состояния (уравнение ползучести) используем в виде степенной зависимости $\dot{\varepsilon}(t) \sim \sigma^{n}(t)$ (по теории течения $\dot{\varepsilon}(t)=B \sigma^{n}(t)$, где $B$ - материальный параметр) скорости деформации удлинения от растягивающего напряжения:

$$
\frac{\dot{\varepsilon}}{\dot{\varepsilon}_{0}}=\left[\frac{\sigma}{\sigma_{0}}\right]^{n},
$$

где начальные значения $\sigma_{0}$ и $\dot{\varepsilon}_{0}$ (скорость деформации удлинения при установившейся ползучести) измерены в эксперименте; $n$ - материальный параметр. Из соотношения (2) следует, что удлинение цилиндрического образца происходит с увеличением скорости деформации $\dot{\varepsilon}(t)$ и нормального напряжения $\sigma(t)$, следовательно, зависимость $\varepsilon(\sigma)$ описывает процесс деформирования, который сопровождается разупрочнением материала. Из равенства усилий

$$
F=\sigma_{0} s_{0}=\sigma s
$$

следует зависимость

$$
\sigma=\sigma_{0}\left[\frac{d_{0}}{d}\right]^{2}
$$

Из условия неизменности объема цилиндрического образца (материал считаем несжимаемым) следует зависимость 


$$
d=d_{0} \sqrt{\frac{l_{0}}{l}} .
$$

Для описания процесса деформирования, при котором $\left[l-l_{0}\right] / l_{0}>0,05$, используем логарифмическую деформацию

$$
\varepsilon=\ln \frac{l}{l_{0}}
$$

Из соотношений (4), (5) и (6) следует зависимость (закон увеличения растягивающего напряжения при однородном удлинении цилиндрического образца)

$$
\sigma=\sigma_{0} \exp (\varepsilon)
$$

Подстановка соотношения (7) в уравнение (2) с последующим интегрированием обеих частей равенства в пределах от нуля до момента появления шейки приводит к определяющему соотношению (определяет аппроксимирующую кривую ползучести)

$$
\int_{0}^{\varepsilon_{*}} \frac{d \varepsilon}{[\exp (\varepsilon)]^{n}}=\dot{\varepsilon}_{0} t .
$$

Обратим внимание, что номинальное напряжение в (8) учитывается неявно через скорость установившейся ползучести, а именно $\dot{\varepsilon}_{0}=A \sigma_{0}^{n}$, где $A$ - материальный параметр.

\section{4. Результаты и обсуждение}

Рассмотрим пример, в котором используем результаты испытаний (таблица), полученных на цилиндрических образцах с диаметром 5 мм и рабочей базой 25 мм (рис. 1), при этом измерение зависимости удлинения от времени проводилось по положению нижнего захвата (подвижного) относительного верхнего захвата (неподвижного) испытательной установки [6]. Результаты этих испытаний получены в НИИ механики МГУ им. М.В. Ломоносова на установке ИМех-5, предназначенной для испытаний на ползучесть и длительную прочность цилиндрических и трубчатых образцов на одноосное растяжение и совместное растяжение и кручение (усилия задаются независимо друг от друга). Нагрев образца производили в воздушной среде внутри цилиндрической печи (максимальная температура нагрева $\left.850{ }^{\circ} \mathrm{C}\right)$. Нагрев образца длился 120 мин, после этого образец выдерживали без нагрузки 15 мин. Температуру на образце измеряли термопарами. Осевая сила создавалась весом платформы с гирями (процесс нагружения длился 1-2 мин). Система измерений представлена индуктивный датчиком, позволяющим измерить текущее удлинение образца по положению в пространстве подвижного захвата относительно неподвижного.

Механические характеристики ползучести титанового сплава ВТ5 при $600{ }^{\circ} \mathrm{C}$

\begin{tabular}{|c|c|c|c|c|c|}
\hline Номер опыта & $\sigma_{0}, \mathrm{MПа}$ & $\dot{\varepsilon}_{0}, \mathrm{ч}^{-1}$ & $\varepsilon_{*}$ & $t_{*}$ ч & $t_{*}$ ч \\
\hline 1 & 75 & 0,01 & 0,47 & 18,9 & 19,7 \\
\hline 2 & 150 & 0,05 & 0,23 & 3,8 & 4,4 \\
\hline 3 & 225 & 0,12 & 0,23 & 1,4 & 1,4 \\
\hline
\end{tabular}


Приведены следующие механические характеристики: $\dot{\varepsilon}_{0}\left(\sigma_{0}\right)$ - зависимость скорости деформации удлинения при установившейся ползучести от номинального напряжения; $\varepsilon_{*}$ и $t_{*}$ - деформация удлинения и момент времени появления шейки (в модели не используется); $t_{* *}-$ время в момент разрушения. Из анализа данных следует, что увеличение $\sigma_{0}$ приводит к увеличению $\dot{\varepsilon}_{0}$ и уменьшению $t_{* *}$, при этом процесс однородного деформирования происходил при относительно больших значениях логарифмической деформации $\varepsilon_{*}=0,23 \div 0,47$. Минимальная погрешность $\Delta=\sum\left|\lg \left(\varepsilon^{\text {approx }} / \varepsilon^{\text {exper }}\right)\right| \rightarrow \min$ расхождения расчетных и экспериментальных кривых ползучести соответствует материальному параметру $n=3,5$, при котором наблюдается удовлетворительное согласование предложенной модели с экспериментальными данными (рис. 2).

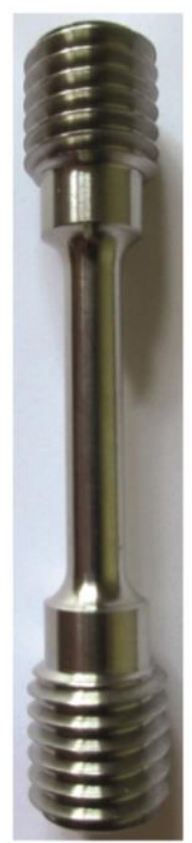

Рис. 1. Цилиндрический образец для механических испытаний на одноосное растяжение

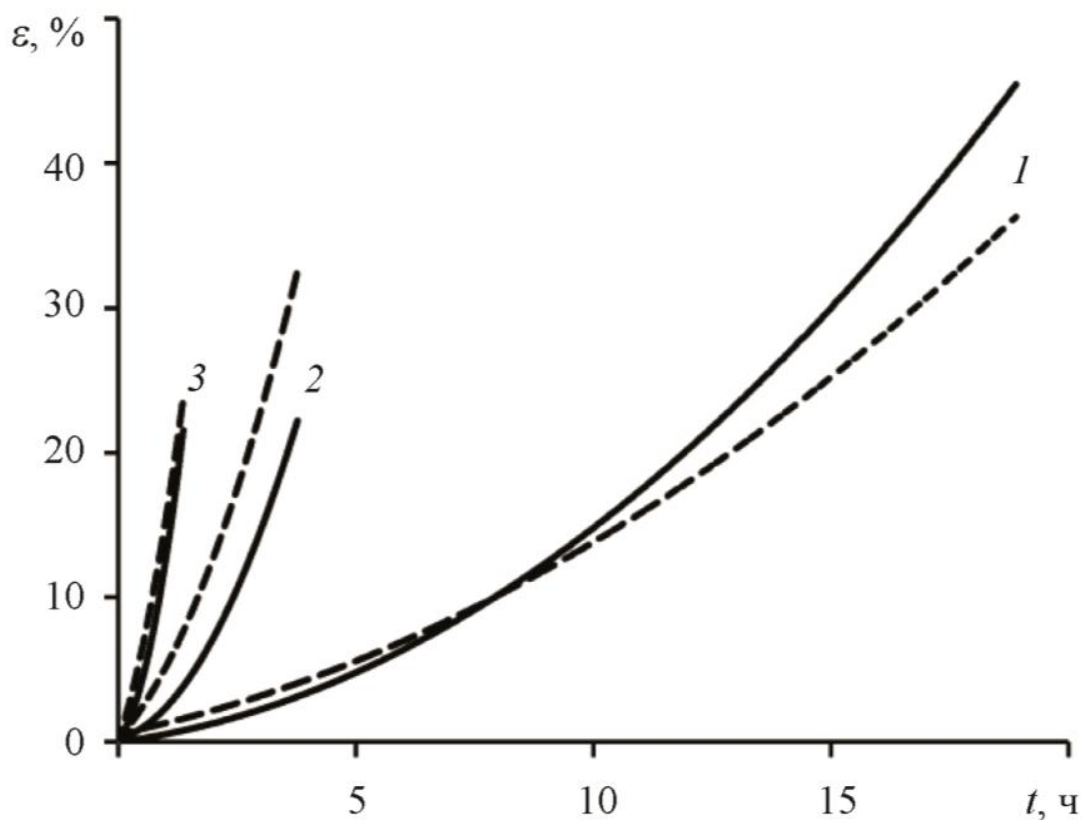

Рис. 2. Зависимости логарифмической деформации ползучести от времени при разных номинальных напряжениях: 1 - 75 МПа; 2 - $150 \mathrm{MПа;} 3$ - 225 МПа, (сплошные линии - опытные данные, штриховые линии аппроксимации при $n=3,5$ )

\section{Литература}

1. Назаров В. В., Лепешкин А. Р. Особенности формоизменения и разрушения прямоугольной пластины с круговым отверстием // Известия ВолгГТУ. Проблемы материаловедения, сварки и прочности в машиностроении. - 2016. - № 2. - С. 75-78.

2. Derevyagina L. S., Panin V. E. and Gordienko A. I. Self-organization of plastic shears in localized deformation macrobands in the neck of high-strength polycrystals, its role in material fracture under uniaxial tension // Physical Mesomechanics. - 2008. - Vol. 11, iss. 1-2. - P. 51-62. DOI: 10.1016/j.physme.2008.03.006.

3. Ахметзянов М. Х., Албаут Г. Н., Барышников В. Н. Исследование напряженнодеформированного состояния в шейке плоских металлических образцов при растяжении методом фотоупругих покрытий // Заводская лаборатория. Диагностика материалов. 2004. - № 8. - C. 41-51. 
4. Локощенко А. М., Терауд В. В. Исследование методом фоторегистрации локализации деформации в цилиндрических образцах при высокотемпературной ползучести // Деформация и разрушение материалов. - 2013. - № 11. - С. 43-46.

5. Моделирование процесса ползучести на основе аппроксимации экспериментальных данных / К. А. Агахи, В. Н. Кузнецов, А. М. Локощенко, В. К. Ковальков, Л. В. Фомин // Машиностроение и инженерное образование. - 2011. - № 2. - С. 52-57.

6. Ковальков В. К., Назаров В. В., Новотный С. В. Методика проведения высокотемпературных испытаний при сложном напряженном состоянии // Заводская лаборатория. Диагностика материалов. - 2006. - № 4. - С. 42-44. 